\title{
Enhancing photoassociation rates by non-resonant light control of shape resonances
}

\author{
Rosario González-Férez ${ }^{1}$ and Christiane P. Koch ${ }^{2}$ \\ ${ }^{1}$ Instituto Carlos I de Física Teórica y Computacional and Departamento de Física Atómica, \\ Molecular y Nuclear, Universidad de Granada, 18071 Granada, Spain \\ ${ }^{2}$ Theoretische Physik, Universität Kassel, Heinrich-Plett-Str. 40, 34132 Kassel, Germany
}

(Dated: July 10, 2018)

\begin{abstract}
Photoassociation, assembling molecules from atoms using laser light, is limited by the low density of atom pairs at sufficiently short interatomic separations. Here, we show that non-resonant light with intensities of the order of $10^{10} \mathrm{~W} / \mathrm{cm}^{2}$ modifies the thermal cloud of atoms, enhancing the Boltzmann weight of shape resonances and pushing scattering states below the dissociation limit. This leads to an enhancement of photoassociation rates by several orders of magnitude and opens the way to significantly larger numbers of ground state molecules in a thermal ensemble than achieved so far.
\end{abstract}

\section{INTRODUCTION}

The formation of ultracold molecules $(T \leq 100 \mu \mathrm{K})$ has experienced rapid progress over recent years [1]. At ultralow temperatures, extremely precise control over the molecules' dynamics can be exerted. This allows for transferring molecules into a single quantum state and even for controlling their reactivity [2]. Their rich internal structure makes them sensitive probes in precision measurements of fundamental constants [3]. Possibly strong dipolar interactions facilitate their use in quantum computation and quantum simulation [4].

To produce molecular samples at ultralow temperatures, molecules are typically assembled from ultracold atoms using magnetic-field controlled Feshbach resonances or laser light. As demonstrated in a series of spectacular experiments, ultracold molecules in their internal ground state are obtained by subsequently applying Stimulated Raman Adiabatic Passage (STIRAP) [5-7] or broadband vibrational cooling [8]. However, in all these experiments, the number of ground state molecules that can be produced has so far been limited to about $10^{4}$. When the molecules are formed by magnetoassociation, this small number is explained by the very low temperature, of the order of hundreds of nano-Kelvin, and corresponding high phase space density, attained by e.g. evaporative cooling. In the case of photoassociation, usually carried out in magneto-optical traps holding up to $10^{10}$ atoms at temperatures between $1-100 \mu \mathrm{K}$ and a phase space density many orders of magnitude lower, only a small fraction of atom pairs resides at sufficiently short interatomic separations to be photoassociated. The number of ground state molecules produced directly by photoassociation has thus also been limited to about $10^{4}[9]$. A significant increase of the photoassociation rate is the problem that we address here.

To this end, we suggest to control shape resonances using non-resonant light to enhance photoassociation rates. Non-resonant light couples to the anisotropic polarizability of an atom pair, shifting the position of shape resonances to lower energies [13] and increasing the resonance's thermal weight in an ultracold trap. Be- low we demonstrate an enhancement by three orders of magnitude in the photoassociation rate of strontium, a molecule which is currently attracting considerable attention $[11,12,14,15]$. Photoassociation relies only on the presence of optical transitions which usually are abundant. Shape resonances are ubiquitous for diatomics. The light-matter coupling via the anisotropic polarizability is of universal character, independent of a particular energy level structure, frequency of the light, or presence of a permanent dipole moment. Therefore our findings are easily extended to molecules other than $\mathrm{Sr}_{2}$.

Our approach is related to Feshbach-optimized photoassociation [16] which also employs a scattering resonance to increase the number of atom pairs that are quasi-trapped at short interatomic separations. Instead of tuning an existing resonance, one could also use resonances induced by an external field, for example, electricfield induced resonances for polar molecules [17, 18]. However, the electric fields required to achieve a significant enhancement (of about $1.3 \mathrm{MV} / \mathrm{cm}$ in the most favorable case) are currently experimentally unfeasible [19]. Unlike Feshbach-optimized photoassociation, shape resonance control does not require the presence of a hyperfine manifold of the atoms. Shape resonances occur naturally for partial waves with $l>0$ when a scattering state becomes trapped behind the centrifugal barrier. Since shape resonances are quasi-bound states, they have very favorable free-to-bound overlaps required for efficient photoassociation; cf. the blue line in Fig. 1 (left). In some fortitious cases such as $\mathrm{Rb}_{2}[20,21]$ or $\mathrm{Cs}_{2}$, the energy of a shape resonance almost matches the trap temperature, and large photoassociation rates are observed. Typically, however, the lowest energies at which shape resonances occur are far from the trap temperature, and their thermal weight is very small. In this case, photoassociation is limited by the atom pair density $\rho(R)$ at interatomic separations $R \lesssim 200 \mathrm{a}_{0}$ [22]; i.e., it addresses mostly $s$-wave atom pairs for which free-to-bound Franck-Condon factors are very small, cf. the grey line in Fig. 1 (left). This can be changed by applying a strong non-resonant field prior to and during photoassociation. 


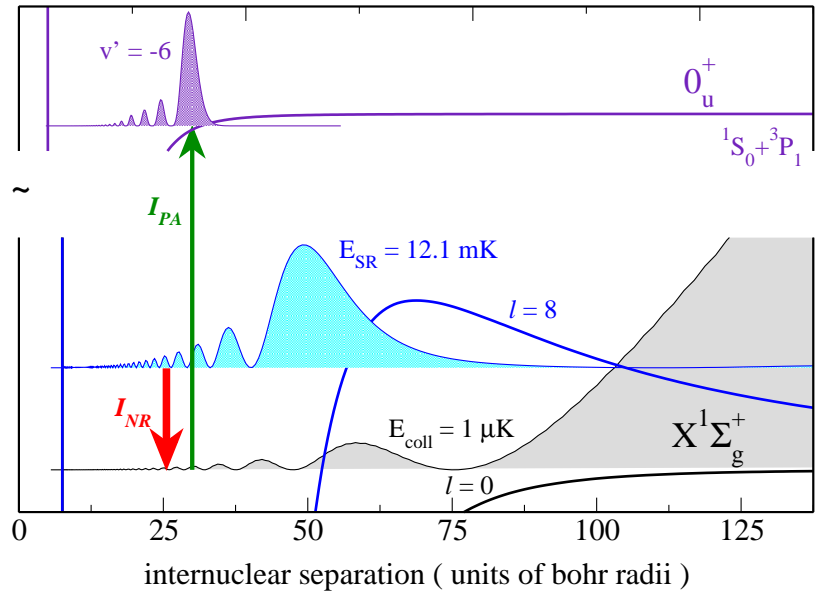

FIG. 1: (Color online) left: The photoassociation efficiency is determined by the free-bound Franck-Condon overlap between the scattering states and weakly bound excited state levels (purple line), shown here for photoassociation of ${ }^{88} \mathrm{Sr}_{2}$ molecules near an intercombination line transition [10-12]. For $s$-waves (grey line, scaled by a factor of $10^{6}$ compared to the blue line), the probability density at short internuclear distances is very small, leading to low photoassociation efficiency. A shape resonance (blue line corresponding to a field-free rotational quantum number $l=8$ ) is a quasi-bound state trapped behind the rotational barrier. Typically, its energy, $E_{S R}$, is too high for the shape resonance to carry any significant thermal weight in an ultracold cloud. Here we suggest to employ a strong non-resonant field (red arrow) which leads to rotational hybridization of the scattering states, effectively moving the position of the shape resonance to lower collision energies, $E_{\text {coll }}$, and increasing the thermal weight of the shape resonance.

\section{THEORETICAL FRAMEWORK}

We assume the non-resonant light, with intensity $I$, and a photoassociation laser, with intensity $I_{P A}$, to be linearly polarized along the $z$-axis of the laboratory fixed frame. Then, in the body fixed frame, the interaction of a pair of atoms with these two fields is described by the Hamiltonian

$$
H=\left(\begin{array}{cc}
H_{g} & -\frac{D(R)}{2} \sqrt{\frac{I_{P A}}{2 \epsilon_{o} c}} \cos \theta \\
-\frac{D(R)}{2} \sqrt{\frac{I_{P A}}{2 \epsilon_{o} c}} \cos \theta & H_{e}+\Delta_{P A}
\end{array}\right)
$$

with

$$
H_{j}=T+V_{j}(R)+\frac{\vec{L}^{2}}{2 \mu R^{2}}-\frac{I}{2 \epsilon_{0} c}\left[\Delta \alpha^{j}(R) \cos ^{2} \theta+\alpha_{\perp}^{j}(R)\right]
$$

and $j=g, e$ for the electronic ground and excited state, respectively. $\Delta_{P A}=\hbar\left(\omega_{P A}-\omega_{a t}\right)$ denotes the detuning of the photoassociation laser from the atomic resonance, $\epsilon_{0}$ and $c$ are the electric vacuum permittivity and speed of light. The photoassociation laser interacts with the transition dipole moment, $D(R)$, coupling the electronic ground state to an electronically excited state. $V_{g}(R)$ and $V_{e}(R)$ denote the respective potential energy curves and $T_{R}$ and $\vec{L}^{2} / 2 \mu R^{2}$ are the vibrational and rotational kinetic energies. The non-resonant light intensity couples to the polarizability anisotropy, $\Delta \alpha^{j}(R)=\alpha_{\|}^{j}(R)-\alpha_{\perp}^{j}(R)(j=g, e)$, where $\alpha_{\| / \perp}^{j}(R)$ are the parallel and perpendicular molecular polarizability components. The behaviour at large internuclear distances is well approximated by Silberstein's formula in terms of atomic polarizabilities $[23,24]$. We consider ${ }^{88} \mathrm{Sr}$ atoms trapped at a temperature of $1 \mu \mathrm{K}$. The potential energy curve for the electronic ground state was taken from Ref. [25] and adjusted to yield a scattering length of $a_{S}=-2 a_{0}$ [35]. The potential energy curves for the electronically excited $\mathrm{A}^{1} \Sigma_{u}^{+}, \mathrm{c}^{3} \Pi_{u}$, and $\mathrm{a}^{3} \Sigma_{u}^{+}$manifold, spin-orbit coupling functions and transition dipole matrix elements are found in Ref. [14]. We investigate photoassociation into the lowest level below the ${ }^{1} S+{ }^{3} P$ asymptote that was previously observed $\left(v^{\prime}=-6\right)[10]$. This level is well represented by an adiabatic potential energy curve $0_{u}^{+}$neglecting resonant spinorbit coupling [15]. Its spontaneous emission lifetime was calculated to be $\gamma_{v^{\prime}=-6}=7.6 \mu \mathrm{s}$ [15]. The atomic polarizabilities for ground and excited state are taken from Refs. [26, 27]. The Hamiltonian (1) is represented by a mapped Fourier grid for the radial part and a basis set expansion in terms of Legendre polynomials for the angular part, taking advantage of the magnetic quantum number $m$ being conserved. About $N_{R} \approx 2000$ radial grid points and $l_{\max }=20$ are required to converge the calculations for $T=1 \mu \mathrm{K}$ and $I \leq 1.5 \times 10^{10} \mathrm{~W} / \mathrm{cm}^{2}$.

Diagonalizing the Hamiltonian (1) for $I_{P A}=0$ yields the bound levels and continuum states of the ground and excited electronic states in the presence of the nonresonant field. For $I \neq 0$, only the magnetic quantum number is conserved. The non-resonant field mixes different partial waves of the same parity and $l$ and $l^{\prime}$ are not good quantum number any more. For the sake of simplicity, we will label the field-dressed states by the field-free quantum numbers $l, m$, and $v^{\prime}, l^{\prime}, m^{\prime}$, adding a tilde to $l, v^{\prime}$ and $l^{\prime}$ to indicate that they are labels not quantum numbers. Note that for bound states, the fielddressed levels $\tilde{v}^{\prime}, \tilde{l}^{\prime}$ are adiabatically connected to the field-free quantum numbers $v^{\prime}, l^{\prime}$ even for very large intensities. The hybridization of the angular motion of the field-dressed wave functions is analyzed in terms of the rotational weights of the field-free partial waves,

$$
c_{l}=\int d E \int d R \int d \cos \theta \psi_{\tilde{l}, 0}^{g}\left(R, \theta ; E_{S R}\right) \psi_{l, 0}^{g}(R, \theta ; E),
$$

where $\psi_{\tilde{l}, 0}^{g}\left(R, \theta ; E_{S R}\right)=\left\langle R, \theta \mid \psi_{\tilde{l} m=0}^{g}\left(E_{S R}\right)\right\rangle$, and $\psi_{l, 0}^{g}(R, \theta ; E)$ are the field-free scattering wavefunctions for partial wave $l$. The photoassociation rate coefficient in the presence of the non-resonant field is determined from the excited state bound levels and ground state continuum states, $\left|\psi_{\tilde{v}^{\prime} \tilde{l}^{\prime} m^{\prime}}^{e}\right\rangle$ and $\left|\psi_{\tilde{l} m}^{g}(E)\right\rangle$ with $\left|\psi_{\tilde{l} m}^{g}(E)\right\rangle$ normalized with respect to energy [29].

For an ensemble of atom pairs with a Maxwell- 
Boltzmann velocity distribution, employing an isolated photoassociation resonance, the photoassociation rate co- effcient is given by [28]

$$
K_{\tilde{v}^{\prime}}\left(I_{P A}, \omega_{P A}, I, T\right)=\frac{k_{B} T}{h Q_{T}} \int_{0}^{\infty} \sum_{\tilde{l}=0}^{\infty} \sum_{\tilde{l}^{\prime}=0}^{\infty} e^{-\frac{E}{k_{B} T}} \frac{\gamma_{\tilde{v}^{\prime} \tilde{l}^{\prime} m^{\prime}} \gamma_{s}\left(I_{P A}, \tilde{v}^{\prime}, \tilde{l}^{\prime}, m^{\prime}, E, \tilde{l}, m, I\right)}{\left(E-\Delta_{\tilde{v}^{\prime} \tilde{l}^{\prime} m^{\prime}}\right)^{2}+(\gamma / 2)^{2}} \frac{d E}{k_{B} T}
$$

with $\gamma_{\tilde{v}^{\prime} \tilde{l}^{\prime} m^{\prime}}$ the spontaneous decay rate of level $\tilde{v}^{\prime} \tilde{l}^{\prime} m^{\prime}, \quad$ approximated by $\gamma_{v^{\prime}=-6, l^{\prime}=0, m^{\prime}=0,} \gamma_{s} \equiv$ $\gamma_{s}\left(I_{P A}, \tilde{v}^{\prime}, \tilde{l}^{\prime}, m^{\prime}, E, \tilde{l}, m, I\right)$ the stimulated emission rate, and $\gamma$ the total width, $\gamma=\gamma_{\tilde{v}^{\prime} \tilde{l}^{\prime} m^{\prime}}+\gamma_{s} . \quad Q_{T}=$ $\left(2 \pi \mu k_{B} T / h^{2}\right)^{3 / 2}$ denotes the translational partition function, and $\Delta_{\tilde{v}^{\prime} \tilde{l}^{\prime} m^{\prime}}$ the detuning, $\Delta_{\tilde{v}^{\prime} \tilde{l}^{\prime} m^{\prime}}=E_{\tilde{v}^{\prime} \tilde{l}^{\prime} m^{\prime}}-\hbar \omega_{P A}$. Since ${ }^{88} \mathrm{Sr}_{2}$ is a bosonic molecule with zero nuclear spin, the sum in Eq. (3) runs solely over even partial waves $\tilde{l}$ in the electronic ground state. We consider only $m=m^{\prime}=0$ in Eq. (3), for which the effect of the non-resonant field is largest. The actual photoassociation rate will be slightly higher than predicted here due to the neglected contributions from terms with $m \neq 0$ to the sum of Eq. (3). For weak photoassociation intensities, the stimulated emission rate can be approximated by Fermi's golden rule,

$$
\begin{aligned}
& \gamma_{s}\left(I_{P A}, \tilde{v}^{\prime}, \tilde{l}^{\prime}, m^{\prime}, E, \tilde{l}, m, I\right) \approx \\
& \frac{4 \pi^{2} I_{P A}}{c}\left|\left\langle\psi_{\tilde{v}^{\prime} \tilde{l}^{\prime} m^{\prime}}^{e}|D(R) \cos \theta| \psi_{\tilde{l} m}^{g}(E)\right\rangle\right|^{2},
\end{aligned}
$$

i.e., by an integral over $R$ and $\theta$, ensuring the correct selection rules.

\section{RESULTS: PHOTOASSOCIATION OF SR}

The photoassociation rate, $K_{\tilde{v}^{\prime}}\left(I_{P A}, \omega_{P A}, I, T\right)$, is shown in Fig. 2 for $\tilde{v}^{\prime}=-6$ and weak (top) and strong (bottom) non-resonant fields. The black solid line shows the typical rotational progression of a photoassociation spectrum for $I=0$. The largest peak is observed for $l^{\prime}=1$, reflecting the pure $s$-wave character of the thermal cloud at $1 \mu \mathrm{K}$. A weak non-resonant field (Fig. 2, top) shifts the photoassociation peaks. The largest shift occurs for $\tilde{l}^{\prime}=1$. For the optical lattice of Ref. [12] with $I \sim 10^{7} \mathrm{~W} / \mathrm{cm}^{2}$, the shift amounts to about $3 \mathrm{MHz}$. An increase of the peak heights is only observed for $\tilde{l}^{\prime}=3$ and $\tilde{l}^{\prime}=5$. It is rationalized in terms of rotational hybridization in the excited state levels. Generally, bound levels are more strongly affected by the non-resonant field than scattering states. The $\tilde{l}^{\prime}=3$ and $\tilde{l}^{\prime}=5$ states have thus some contribution from the $l^{\prime}=1$ partial wave which renders photoassociation from $s$-wave atom pairs into the field-dressed $\tilde{l}^{\prime}=3$ and $\tilde{l}^{\prime}=5$ bound state allowed, enhancing the corresponding photoassociation peak. While

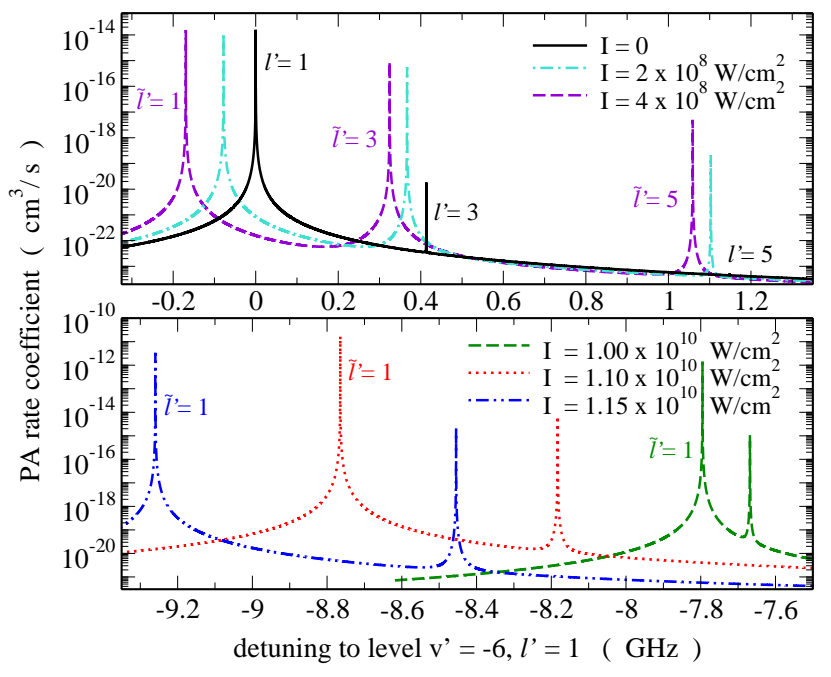

FIG. 2: (Color online) photoassociation lines (absorption rate coefficient) for photoassociation into $\tilde{v}^{\prime}=-6$ for weak (top) and strong (bottom) non-resonant fields $I$. The intensity of the photoassociation laser is set to $I_{P A}=1 \mathrm{~W} / \mathrm{cm}^{2}$, and the trap temperature to $T=1 \mu \mathrm{K}$.

this increase amounts to several orders of magnitude, the total photoassociation rate for $\tilde{l}^{\prime}=3$ and $\tilde{l}^{\prime}=5$ is still smaller than the $l^{\prime}=1$ photoassociation rate without non-resonant field.

At high non-resonant light intensities (Fig. 2, bottom), the rotational progression observed for $I=0$ is replaced by the pendular progression. More remarkably, a large increase of the $\tilde{l}^{\prime}=1$ peak, amounting to about three orders of magnitude compared to the field-free $l^{\prime}=1$ photoassociation rate, is observed in Fig. 2 for non-resonant field intensities of the order of $10^{10} \mathrm{~W} / \mathrm{cm}^{2}$. For $T=1 \mu \mathrm{K}$, the $\tilde{l}=12$ shape resonance acquires its highest thermal weight close to $I \sim 10^{10} \mathrm{~W} / \mathrm{cm}^{2}$, cf. Fig. 3 showing the resonance position as a function of non-resonant field intensity. The remarkable enhancement of the $\tilde{l}^{\prime}=1$ photoassociation rate in Fig. 2 is thus related to the fielddressed $\tilde{l}=12$ shape resonance. This is further analyzed by inspection of the rotational weights, Eq. (2), of the field-dressed resonance wavefunction shown in Fig. 4. The lower (upper) panel of Fig. 4 displays the components for low (high) $l$. Note that the coupling mixes only partial waves of the same parity. For $I=0$, the resonance wavefunctions are pure $l=4, l=8$ and $l=12$ states, 


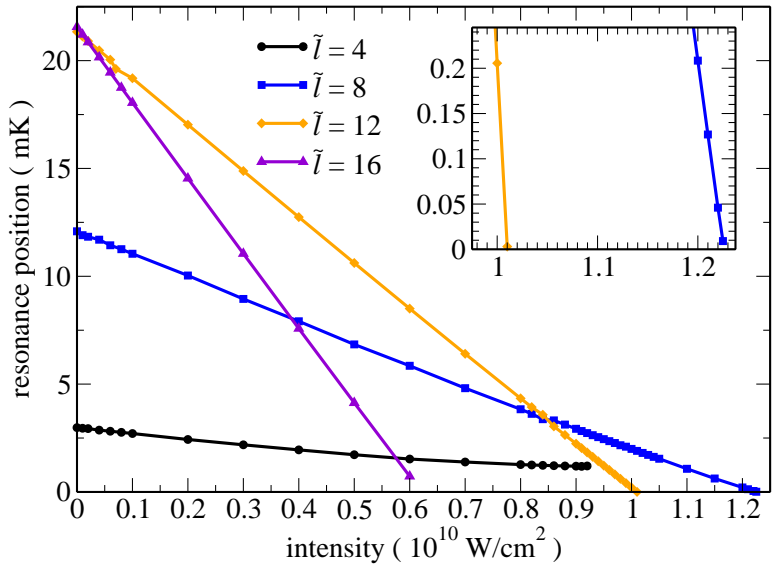

FIG. 3: Energy of the $\tilde{l}=4, \tilde{l}=8, \tilde{l}=12$ and $\tilde{l}=16$ shape resonances vs non-resonant field intensity.

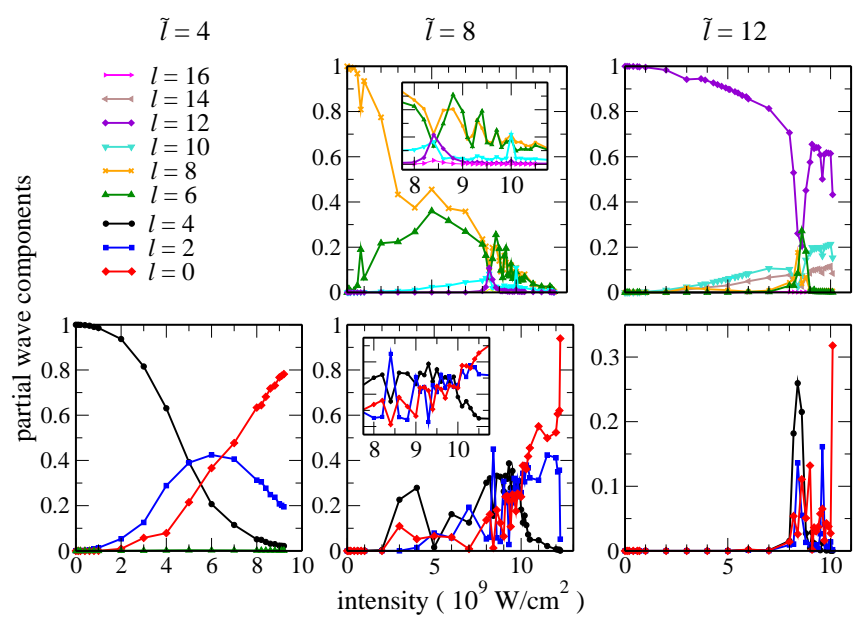

FIG. 4: (Color online) Contribution of different partial waves $l$ to the resonance wavefunction for the $\tilde{l}=4,8,12$ shape resonances as a function of the non-resonant intensity. The low- $l$ contributions are shown in the lower panel, the high- $l$ contributions in the upper panel. For high non-resonant field intensities, all resonance wavefunctions acquire predominantly $s$-wave character.

respectively. As the non-resonant field is switched on, a substantial amount of first $l-2$ and then $l-4$ is mixed in to the field-free states. For large non-resonant field intensities, the trend of mixing in lower- $l$ components is observed all the way down to $l=0$ (red diamonds).Thus, photoassociation from the field-dressed resonance wave function into $\tilde{l}^{\prime}=1$ becomes possible for large $I$, explaining the large enhancement of photoassociation into an excited state level with $\tilde{l}^{\prime}=1$ observed in the lower panel of Fig. 2.

While the dependence on the non-resonant intensity in Fig. 4 is smooth for the $\tilde{l}=4$ resonance, discontinuous behavior is observed for $\tilde{l}=8, \tilde{l}=12$. Each of the features in the apparantly complicated dependence for $\tilde{l}=8, \tilde{l}=12$ can be rationalized in terms of nonadiabatic behavior of interacting resonances: The first

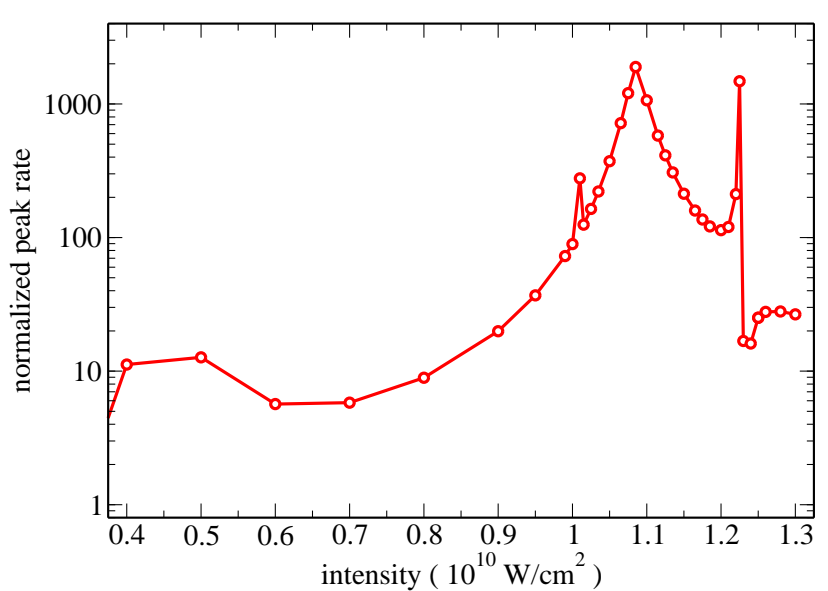

FIG. 5: (Color online) $\tilde{l}^{\prime}=1$ peak in the presence of the field normalized with respect to the largest field-free peak (with $\left.l^{\prime}=1\right)$ in the rate coefficients for photoassociation into $\tilde{v}^{\prime}=$ $-6(T=1 \mu \mathrm{K})$. Enhancement of the photoassociation rate of about three orders of magnitude is found when the position of a shape resonance comes close to the trap temperature.

kink in the dependence of the $\tilde{l}=8$ resonance is observed at $8 \times 10^{8} \mathrm{~W} / \mathrm{cm}^{2}$. It is due to the interaction with a $\tilde{l}=6$ shape resonance [36]. A second discontinuous feature for $\tilde{l}=8$ is observed around $4 \times 10^{9} \mathrm{~W} / \mathrm{cm}^{2}$. It is caused by the interaction of the $\tilde{l}=8$ and $\tilde{l}=16$ resonances close to their crossing, see Fig. 3. The most striking nonadiabatic features occur for both $\tilde{l}=8$ and $\tilde{l}=12$ resonances between $8 \times 10^{9} \mathrm{~W} / \mathrm{cm}^{2}$ and $1 \times 10^{10} \mathrm{~W} / \mathrm{cm}^{2}$. It is due to the non-adiabatic interaction between these two resonances which cross near $8.5 \times 10^{9} \mathrm{~W} / \mathrm{cm}^{2}$ and with other scattering states, introducing a strong mixing of the resonance wavefunctions. The non-adiabatic behavior of the shape resonances observed in Fig. 4 underlines the requirement to treat the fully coupled rotationalvibrational dynamics.

The largest increase of the $\tilde{l}^{\prime}=1$ peak in Fig. 2 is found for $I=1.10 \times 10^{10} \mathrm{~W} / \mathrm{cm}^{2}$ (red dotted curve). A detailed analysis of the increase as a function of the non-resonant light intensity is presented in Fig. 5 which shows the height of the $\tilde{l}^{\prime}=1$ peak normalized with respect to the largest field-free $\left(l^{\prime}=1\right)$ peak. Most importantly, Fig. 5 demonstrates an enhancement of the photoassociation rate between two to three orders of magnitude over a broad range of non-resonant field intensities. The narrow peaks at $I=1.01 \times 10^{10} \mathrm{~W} / \mathrm{cm}^{2}$ and $I=1.225 \times 10^{10} \mathrm{~W} / \mathrm{cm}^{2}$ are due to the $\tilde{l}=12$ and $\tilde{l}=8$ shape resonances, cf. Fig. 3. These peaks reflect how the shape resonances pass through the energy window that corresponds to the atomic cloud's thermal width. The broad features are attributed to regular scattering states which are also affected by the nonresonant field. For sufficiently large $I$, a scattering state is pushed below the dissociation threshold (without symmetry restriction, an even parity state is followed by an odd parity state) [18]. Before this happens, the scat- 


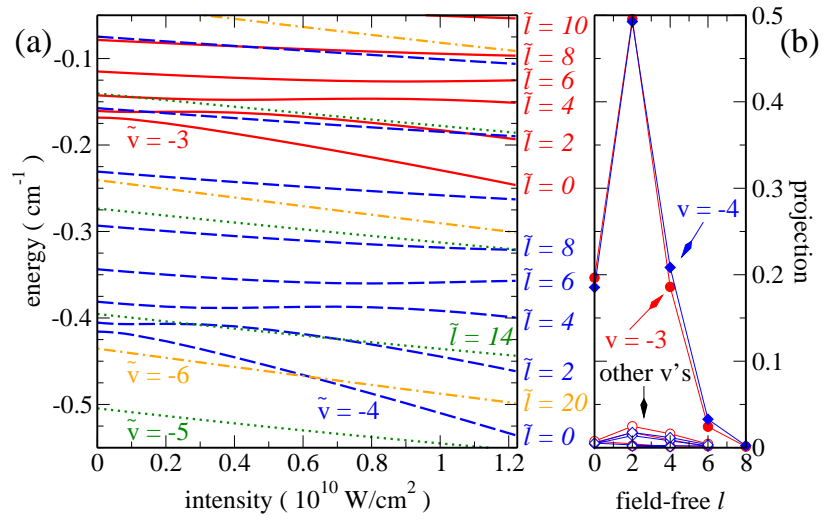

FIG. 6: (Color online) Energy levels (a) and projections (b) of the hybridized $\tilde{v}^{\prime \prime}=-3, \tilde{l}^{\prime \prime}=0$ (red) and $\tilde{v}^{\prime \prime}=$ $-4, \tilde{l}^{\prime \prime}=0$ (blue) ground state levels for adiabatic (a) or sudden (b) transfer into their field-free counterparts. For a slow switch-off, non-adiabatic transitions are not expected and all molecules are obtained with $l^{\prime \prime}=0$ (a), while for a sudden switch-off, about $20 \%$ of the molecules have $l^{\prime \prime}=0$ and about $50 \% l^{\prime \prime}=2$.

tering wave function is compressed to short internuclear distances, yielding an increase in the photoassociation rate. This is observed in Fig. 5 for intensities around $I=1.085 \times 10^{10} \mathrm{~W} / \mathrm{cm}^{2}$. The corresponding state becomes bound at $I \approx 1.22 \times 10^{10} \mathrm{~W} / \mathrm{cm}^{2}$. Also the peak near $I=5 \times 10^{9} \mathrm{~W} / \mathrm{cm}^{2}$ is caused by a state being pushed below the dissociation threshold. However, the enhancement is much larger for $I=1.085 \times 10^{10} \mathrm{~W} / \mathrm{cm}^{2}$ since this state becomes bound inbetween two shape resonances.

The sharp features of the peak enhancement shown in Fig. 5 clearly reflect the $l=0$ rotational weight of the $\tilde{l}=8$ and $\tilde{l}=12$ shape resonances. The small peak at $1.01 \mathrm{~W} / \mathrm{cm}^{2}$ is connected to the $\left|c_{l=0}\right|^{2}$ weight of the $\tilde{l}=12$ shape resonance; and the sharp rise and drop of the normalized peak rate in Fig. 5 at $1.225 \mathrm{~W} / \mathrm{cm}^{2}$ reflects the behavior of the $\left|c_{l=0}\right|^{2}$ weight for $\tilde{l}=8$ shape resonance before it becomes bound. The $\tilde{l}=16$ shape resonance, also shown in Fig. 3 suffers almost no hybridization of its angular motion, with $\left|c_{l=16}\right|^{2}$ remaining as high as 0.9 for $I=6.1 \times 10^{9} \mathrm{~W} / \mathrm{cm}^{2}$, just before the resonance becomes bound. Therefore the dipole coupling to $\tilde{v}^{\prime}=-6, \tilde{l}=1$ remains rather small, and no enhancement due to $\tilde{l}=16$ shape resonance is observed in Fig. 5.

In absolute numbers, the maximum peak in Fig. 5 corresponds to a rate of $3 \times 10^{-11} \mathrm{~cm}^{3} / \mathrm{s}$, comparable to the photoassociation rates of $\mathrm{Cs}_{2}$ molecules in weakly bound levels of the $0_{g}^{-}\left(P_{3 / 2}\right)$ excited state [30]. The important difference to $\mathrm{Cs}_{2}$ is due to the predominantly $1 / R^{6}$ longrange behavior of the electronically excited state. This allows almost all of the photoassociated molecules to decay into bound ground state levels and efficient subsequent Raman transfer to low lying vibrational states.

The bound states of both ground and excited electronic state are characterized by strong alignment in the presence of the non-resonant field [31]. For example, $\left\langle\cos ^{2} \theta\right\rangle \gtrsim 0.94$ for $I \geq 10^{10} \mathrm{~W} / \mathrm{cm}^{2}$. In the presence of the non-resonant field, the photoassociated molecules spontaneously decay into hybridized levels of the electronic ground state. The branching ratios are calculated from the transition dipole moments [15] of the fielddressed rovibrational states. For $I=1.1 \times 10^{10} \mathrm{~W} / \mathrm{cm}^{2}$, $\tilde{v}^{\prime}=-6, \tilde{l}^{\prime}=1$ shows a branching ratio of $31 \%$ to $\tilde{v}^{\prime \prime}=-3, \tilde{l}^{\prime \prime}=0$ and $43 \%$ to $\tilde{v}^{\prime \prime}=-4, \tilde{l}^{\prime \prime}=0$ compared to field-free branching ratios of $51 \%$ and $41 \%$ for $v^{\prime \prime}=-3, l^{\prime \prime}=0$ and $l^{\prime \prime}=2$, respectively [37]. Once molecules in the electronic ground state are produced, the non-resonant field has to be switched off. If this is done adiabatically, the hybridized ground state levels $\tilde{v}^{\prime \prime}=-3, \tilde{l}^{\prime \prime}=0$ and $\tilde{v}^{\prime \prime}=-4, \tilde{l}^{\prime \prime}=0$ are directly connected with $v^{\prime \prime}=-3, l^{\prime \prime}=0$ and $v^{\prime \prime}=-4, l^{\prime \prime}=0$. A slow switch-off of the non-resonant field transfers these hybridized levels to their field-free counterparts unless avoided level crossings occur. For $\tilde{v}^{\prime \prime}=-3, \tilde{l}^{\prime \prime}=0$ and $\tilde{v}^{\prime \prime}=-4, \tilde{l}^{\prime \prime}=0$, crossings may be found only for very highly excited rotational states from lower vibrational bands, $\tilde{l}^{\prime \prime} \geq 20$, for which the non-adiabatic couplings with $l^{\prime \prime}=0$ are small, cf. Fig. 6(a). A slow switchoff of the non-resonant field thus produces ground state molecules with $v^{\prime \prime}=-3$ or $v^{\prime \prime}=-4$ and $l^{\prime \prime}=0$. If the molecules after spontaneous decay are subject to loss processes in the trap, it may not be possible to switch the field off adiabatically. A sudden switch-off projects the hybridized ground state levels on a superposition of $m^{\prime}=0$ field-free rotational levels of the same vibrational band with about $50 \% l^{\prime}=2,20 \% l^{\prime}=0$, and $20 \%$ $l^{\prime}=4$ components for both $v^{\prime \prime}=-3$ and $v^{\prime \prime}=-4$, cf. Fig. 6(b). Thus, a large part of the molecules is transferred to ground vibrational levels with $l^{\prime \prime}=0$ for both slowly or suddenly switching off the field. The weakly bound ground state levels show large two-photon transition matrix elements for efficient Raman transfer to low-lying rovibrational levels of the electronic ground state [15]. Almost all of the molecules produced by photoassociation can thus be transferred to the rovibrational ground state, in contrast to all previous photoassociation schemes realized experimentally to date.

\section{CONCLUSIONS}

We have shown that the photoassociation rate of $\mathrm{Sr}_{2}$ molecules is enhanced by three orders of magnitude by applying an additional non-resonant field. This enhancement is due to shape resonances and scattering states becoming bound, causing a larger number of atom pairs to be quasi-trapped at sufficiently short interatomic separations to be photoassociated. Since the photoassociation rate is limited by the low pair density at or near the Condon radius [22, 32], applying strong non-resonant light during photoassociation overcomes the main obstacle toward forming larger numbers of molecules. The enhancement of the photoassociation rate is accompanied 
by strong hybridization of the angular motion, which we have fully accounted for in a rigorous treatment of the coupled rovibrational motion.

Prior to photoassociation, the non-resonant field needs to be switched on slowly such that the position of shape resonances and scattering states is shifted adiabatically to lower energies. Then the photoassociation laser addresses a thermal cloud that is modified by the nonresonant light, producing molecules in an electronically excited state. As a specific feature of photoassociation near an intercombination line, the majority of photoassociated molecules decays spontaneously into bound ground state levels rather than re-dissociate [10, 15]. In the presence of the non-resonant field, the spontaneous emission involves hybridized levels. To obtain field-free ground state molecules, the non-resonant field needs to be switched off. Ideally, this is done adiabatically, yielding rotationless molecules in two ground state vibrational levels. Alternatively, a sudded switch-off leads to a superposition of ground state molecules with the three lowest rotational quantum numbers, 0, 2 and 4, occupied.

The required non-resonant field intensities of the order of $10^{10} \mathrm{~W} / \mathrm{cm}^{2}$ can be achieved by employing intra-cavity beams. Assuming a spot size of the order of $10 \mu \mathrm{m}$, intracavity powers of up to $10^{4} \mathrm{~W}$ are needed. Such powers can realistically be produced, for example, by injecting $100 \mathrm{~W}$ from a single mode telecom wavelength fiber laser into a cavity with a power build-up factor of one hundred.

Enhanced photoassociation rates are a crucial prerequisite to increase the number of ground state molecules that can be produced optically. For molecules other than $\mathrm{Sr}_{2}$, our scheme works best for heavy atoms with large polarizabilities and large scattering lengths. Promising candidates include, besides other even-isotope alkalineearth dimers or $\mathrm{Yb}_{2}$, heteronuclear molecules comprised of one alkali atom and one alkaline-earth-like atom such as RbSr or RbYb [33]. For these species, where Feshbach association is either not available or likely not to be feasible [34], non-resonant field control of shape resonances paves the way to an efficient all-optical production of ground state molecules.

\section{Acknowledgments}

We would like to thank Michael Drewsen, Ronnie Kosloff, and Robert Moszynski for fruitful discussions. Financial support by the Spanish project FIS2011-24540 (MICINN) as well as the Grants FQM-2445 and FQM4643 (Junta de Andalucía) is gratefully acknowledged. RGF belongs to the Andalusian research group FQM207.
[1] R. V. Krems, W. C. Stwalley, and B. Friedrich, eds., Cold Molecules: Theory, Experiment, Applications (CRC Press, 2009).

[2] S. Ospelkaus, K.-K. Ni, D. Wang, M. H. G. de Miranda, B. Neyenhuis, G. Quéméner, P. S. Julienne, J. L. Bohn, D. S. Jin, and J. Ye, Science 327, 853 (2010).

[3] T. Zelevinsky, S. Kotochigova, and J. Ye, Phys. Rev. Lett. 100, 043201 (2008).

[4] A. Micheli, G. K. Brennen, and P. Zoller, Nature Phys. 2, 341 (2006).

[5] F. Lang, K. Winkler, C. Strauss, R. Grimm, and J. Hecker Denschlag, Phys. Rev. Lett. 101, 133005 (2008).

[6] K.-K. Ni, S. Ospelkaus, M. H. G. de Miranda, A. Pe'er, B. Neyenhuis, J. J. Zirbel, S. Kotochigova, P. S. Julienne, D. S. Jin, and J. Ye, Science 322, 231 (2008).

[7] J. G. Danzl, E. Haller, M. Gustavsson, M. J. Mark, R. Hart, N. Bouloufa, O. Dulieu, H. Ritsch, and H.-C. Nägerl, Science 321, 1062 (2008).

[8] M. Viteau, A. Chotia, M. Allegrini, N. Bouloufa, O. Dulieu, D. Comparat, and P. Pillet, Science 321, 232 (2008).

[9] J. Deiglmayr, A. Grochola, M. Repp, K. Mörtlbauer, C. Glück, J. Lange, O. Dulieu, R. Wester, and M. Weidemüller, Phys. Rev. Lett. 101, 133004 (2008).

[10] T. Zelevinsky, M. M. Boyd, A. D. Ludlow, T. Ido, J. Ye, R. Ciuryło, P. Naidon, and P. S. Julienne, Phys. Rev. Lett. 96, 203201 (2006).

[11] S. Stellmer, B. Pasquiou, R. Grimm, and F. Schreck, Phys. Rev. Lett. 109, 115302 (2012).

[12] G. Reinaudi, C. B. Osborn, M. McDonald, S. Ko- tochigova, and T. Zelevinsky, Phys. Rev. Lett. 109, 115303 (2012).

[13] R. Ağanoğlu, M. Lemeshko, B. Friedrich, R. GonzálezFérez, and C. P. Koch, arXiv:1105.0761.

[14] W. Skomorowski, F. Pawłowski, C. P. Koch, and R. Moszynski, J. Chem. Phys. 136, 194306 (2012).

[15] W. Skomorowski, R. Moszynski, and C. P. Koch, Phys. Rev. A 85043414 (2012).

[16] P. Pellegrini, M. Gacesa, and R. Côté, Phys. Rev. Lett. 101, 053201 (2008).

[17] R. V. Krems, Phys. Rev. Lett. 96, 123202 (2006).

[18] R. González-Férez and P. Schmelcher, New J. Phys. 11, 055013 (2009).

[19] D. Chakraborty, J. Hazra, and B. Deb, J. Phys. B 44, 095201 (2011).

[20] H. M. J. M. Boesten, C. C. Tsai, B. J. Verhaar, and D. J. Heinzen, Phys. Rev. Lett. 77, 5194 (1996).

[21] H. M. J. M. Boesten, C. C. Tsai, J. R. Gardner, D. J. Heinzen, and B. J. Verhaar, Phys. Rev. A 55, 636 (1997).

[22] C. P. Koch, R. Kosloff, E. Luc-Koenig, F. MasnouSeeuws, and A. Crubellier, J. Phys. B 39, S1017 (2006).

[23] T. G. Heijmen, R. Moszyński, P. E. Wormer, and A. van der Avoird, Mol. Phys. 89, 81 (1996).

[24] L. Jensen, P.-O. Åstrand, A. Osted, J. Kongsted, and K. V. Mikkelsen, J. Chem. Phys. 116, 4001 (2002).

[25] A. Stein, H. Knöckel, and E. Tiemann, Phys. Rev. A 78, 042508 (2008).

[26] D. R. Lide, ed., CRC handbook of chemistry and physics (Taylor and Francis, 2009), 91st ed.

[27] S. G. Porsev, A. D. Ludlow, M. M. Boyd, and J. Ye, 
Phys. Rev. A 78, 032508 (2008).

[28] R. Napolitano, J. Weiner, C. J. Williams, and P. S. Julienne, Phys. Rev. Lett. 73, 1352 (1994).

[29] R. González-Férez, M. Weidemüller, and P. Schmelcher, Phys. Rev. A 76, 023402 (2007).

[30] A. Fioretti, D. Comparat, A. Crubellier, O. Dulieu, F. Masnou-Seeuws, and P. Pillet, Phys. Rev. Lett. 80, 4402 (1998).

[31] B. Friedrich and D. Herschbach, Phys. Rev. Lett. 74, 4623 (1995).

[32] C. P. Koch, R. Kosloff, and F. Masnou-Seeuws, Phys. Rev. A 73, 043409 (2006)

[33] F. Münchow, C. Bruni, M. Madalinski, and A. Görlitz, Phys. Chem. Chem. Phys. 13, 18734 (2011).
[34] D. A. Brue, and J. M. Hutson, Phys. Rev. Lett. 108, 043201 (2012)

[35] The photoassociation results are not affected when varying the scattering lengths from $a_{S}=+4.5 a_{0}$ to $a_{S}=$ $-4.5 a_{0}$.

[36] The $\tilde{l}=6$ shape resonance can be identified by inspection of the scattering wavefunctions. However, it is too diffuse for its energy to be determined with sufficient precision and was therefore omitted from Fig. 3.

[37] The field-free branching ratios differ slightly from those published in Ref. [15] due to the adiabatic approximation for the $0_{u}^{+}$state employed here. 